\title{
Ensayos de eficacia cuarentenaria de tres formulaciones de antimanchas comercializadas en Chile para un proceso simulado de exportación de madera aserrada de Pinus radiata
}

\author{
Quarantine efficacy of three anti-bluestain formulas sold in Chile, in a simulated \\ export process involving sawn Pinus radiata timber
}

ISABEL VIVES, SANDRA IDE, HERNAN PEREDO

Instituto de Silvicultura, Facultad de Ciencias Forestales, Universidad Austral de Chile, Casilla 567, Valdivia,Chile. E-mail: isabelvives@uach.cl, side@uach.cl, hperedo@uach.cl

\section{SUMMARY}

Three anti-sapstain products were tested on sawn Pinus radiata timber in the Eighth as well as in the Tenth Regions of Chile. The fungicide products were Combo Antiblu (a mixture of Antiblu 375 and Antiblu CC), Wolsin FL 12215 and Sinesto B. Results showed that the products were more effective in the Tenth Region than in the Eighth Region. Of the three products tested, Combo Antiblu had the best characteristics in both Regions.

Key words: Pinus radiata, bluestain, anti-sapstain.

\section{RESUMEN}

Se ensayaron tres productos antimancha en madera aserrada de Pinus radiata tanto en la Octava como en la Décima Región de Chile. Los productos fungicidas fueron Combo Antiblu (mezcla de Antiblu 375 y Antiblu CC), Wolsin FL 12215 y Sinesto B. De los resultados obtenidos se pudo comprobar que los productos utilizados fueron más efectivos en la Décima que en la Octava Región. De los tres productos ensayados Combo Antiblu tuvo el mejor resultado en ambas regiones al proteger la madera.

Palabras clave: Pinus radiata, mancha azul, antimanchas.

\section{INTRODUCCION}

Pinus radiata es en la actualidad el principal recurso comercializado por el sector forestal de Chile, demostrado así por los 2.205 millones de dólares exportados en pulpa, papel periódico, madera aserrada, trozas aserrables y pulpables durante el año 2001, entre otros (1), cuyos principales destinos en ese año fueron Estados Unidos $(23,2 \%)$ y Japón $(12,5 \%)$. Ambos países exigen que las maderas importadas ingresen libres de agentes patógenos, siendo uno de los más importantes Ceratocystis pilifera (Fries) C. Moreau, causante de la mancha azul y frente al cual $P$. radiata es altamente susceptible (2).

En la exportación de madera los países de destino exigen a sus proveedores que ésta cumpla con ciertos tratamientos que aseguren el arribo a destino sin plagas ni enfermedades. En ese sentido el tratamiento cuarentenario previene que en la exportación de madera vayan agentes que puedan causar daño si entran a otros países que se supone 
BOSQUE 25(3): 79-87, 2004

Ensayos de eficacia cuarentenaria de tres formulaciones de antimanchas comercializadas en Chile para un proceso...

están libres de ellos, determinando así la efectividad de un producto químico. Por lo tanto, el objetivo del presente trabajo fue detectar el efecto del producto químico sobre un producto ya terminado en donde no hay posibilidad de modificar los procesos de producción anteriores a este momento; por lo tanto, se asume un producto estándar que puede o no transportar inóculo. En este contexto, durante el año 2000 se llevaron a cabo ensayos de laboratorio (realizados en el Laboratorio de Patología Forestal, de la Universidad Austral de Chile), de acuerdo a la Norma ASTM D 444591 (3), para determinar la efectividad de ocho productos antimancha, a tres concentraciones cada uno, sobre $C$. pilifera. Posteriormente se eligieron tres de los ocho productos para montar un ensayo de tratamiento cuarentenario en madera aserrada, con el fin de determinar, a escala comercial, la eficacia de estos tres químicos sobre madera aserrada de $P$. radiata en las Regiones Octava y Décima, zonas de donde proviene la mayor parte de las exportaciones.

Todo esto porque hasta el año 1999 en Chile se usaba, de preferencia, Pentaclorofenato de sodio (PCP-Na) como antimancha, sin embargo, esta situación cambió a partir de ese año, ya que el Servicio Agrícola y Ganadero de Chile (SAG) prohibió el uso de fenoles clorados debido a su alto grado de toxicidad, tanto para el hombre como para el medio ambiente (4). Esto ha obligado a las empresas a buscar productos alternativos, tan efectivos como aquél, lo que significa formulaciones más complejas que aumentan los costos. En los últimos años se ha realizado una gran cantidad de ensayos tendientes a encontrar un producto equivalente al PCP-Na en efectividad y costo, pero menos tóxico.

En la actualidad una parte importante de los aserraderos usa Tribromofenol como antimancha en sus distintas presentaciones: Mad Plus, Antistain P-028 y CS 400. No obstante, las empresas químicas siguen en la búsqueda de nuevos productos o ensayando mezclas con los ingredientes activos ya conocidos, debido a que por su carácter fenólico no se descarta la posibilidad que el Tribromofenol pueda ser también prohibido a futuro (4).

\section{MATERIAL Y METODOS}

Al plantearse éste como un ensayo de tratamiento cuarentenario, interesa conocer si la madera se mancha o no, al tratarla con los productos antimanchas ya mencionados. Sin embargo, se consideró de interés hacer una evaluación del grado de ataque del hongo manchador y la presencia de mohos, debido a que había mercados que tenían cierto rechazo a madera con mohos. Para ello se simularon las condiciones de transporte marítimo que en un promedio de 45 días puede transformar desfavorablemente las características del producto exportado.

En la exportación de madera aserrada es común observar que parte de ella es transportada inmediatamente al puerto para su embarque, mientras otra debe permanecer un tiempo en acopio antes de seguir el procedimiento. Por ello se plantea el ensayo en dos líneas de acción diferentes: una primera que inicia la simulación con siete días de acopio para luego ir al transporte marítimo, y una segunda que inicia con 37 días en acopio (figura 1).

La simulación del transporte marítimo se realiza construyendo una estructura (de madera) que permite cubrir el grupo de paquetes que conforman la línea de acción respectiva con polietileno negro (figura 2), semejante a una bodega de barco (5)

Instalación de ensayos: En cada Región se instaló un ensayo de iguales características, consistente en dos grupos (línea 1 y 2 ) de cinco paquetes cada uno, correspondiendo un paquete por cada producto químico en estudio, que, entre el 17 abril y el 4 de septiembre de 2001 y simulando un viaje marítimo típico, sufrieron esquemas de manipulación diferente. Estos se ubicaron en las canchas de acopio de Aserraderos Cementos Bío-Bío, en Concepción (Octava Región), entre los días 17 de abril y 5 de julio para la línea 1 , y 17 de abril al 3 de agosto para la línea 2, y Aserraderos Paillaco en Valdivia (Décima Región), desde el 17 de mayo al 2 de agosto para la línea 1 y desde el 17 de mayo al 4 de septiembre para la línea 2.

Cada uno de los paquetes estaba conformado por 120 tablas dispuestas en filas de 10, subdividido en dos grupos, según si eran separadas por tablillas o no. Los separadores de $30 \times 4 \mathrm{~mm}$ de ancho $\mathrm{y}$ alto respectivamente se utilizaron para 
BOSQUE 25(3): 79-87, 2004 Ensayos de eficacia cuarentenaria de tres formulaciones de antimanchas comercializadas en Chile para un proceso...

\section{Linea}

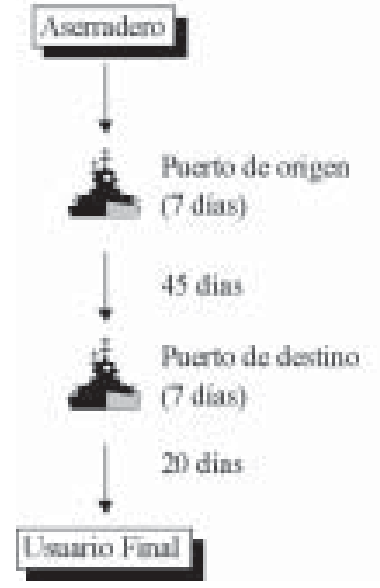

\section{Linea}

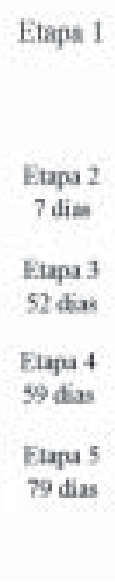

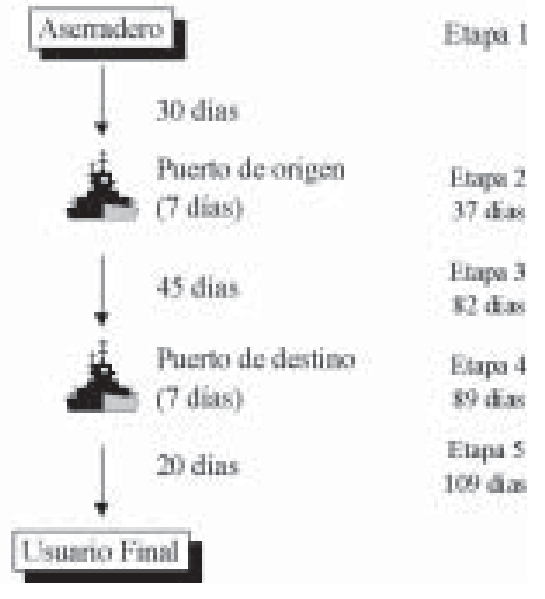

Figura 1. Secuencia de viaje promedio de madera aserrada para un mercado de ultramar. Average travel sequence of timber to an overseas market.

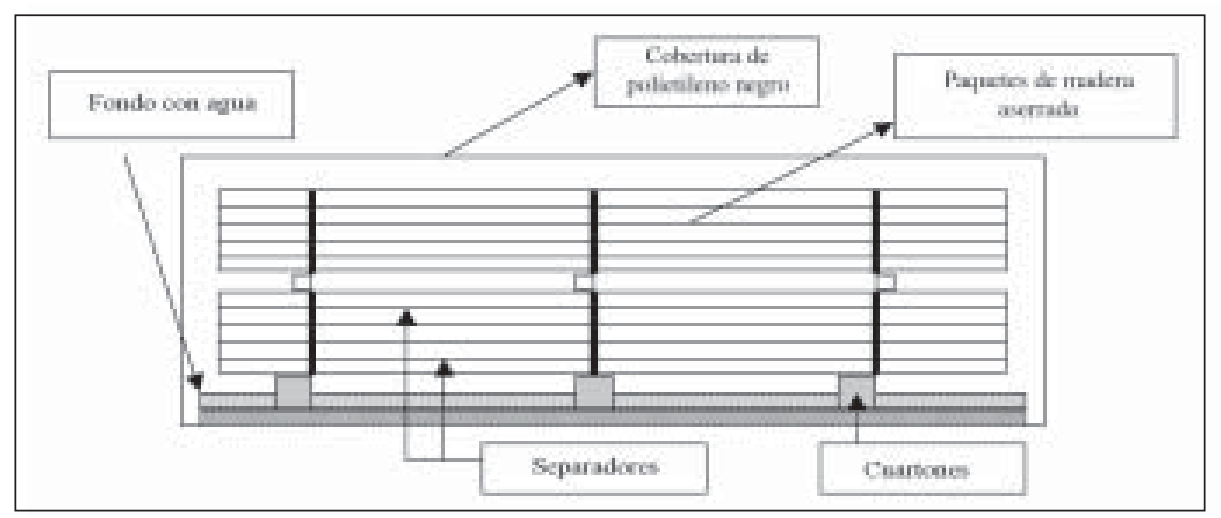

Figura 2. Simulador de bodega de barco para ensayo comercial de madera aserrada (Fuente: Arriagada y Zúñiga, 1996).

Ship store simulator for commercial testing of timber (Source: Arriagada and Zúñiga, 1996).

las primeras seis filas ubicadas en la parte inferior de cada paquete, también se bañaron con el mismo producto químico correspondiente al paquete (figura 2).

Considerando la diferencia en el tipo de productos de los aserraderos, las piezas de madera de los paquetes utilizados en la Octava Región tenían dimensiones de $24 \times 102 \times 4.000 \mathrm{~mm}$, a diferencia de las utilizadas en la Décima Región, cuyas dimensiones eran de $25 \times 100 \times 3.200 \mathrm{~mm}$.

Fungicidas: Las Empresas Hickson Quimetal Ltda. y Basf Chile, representantes para Chile de Combo
Antiblu, Wolsin y Sinesto, respectivamente, fueron las encargadas de realizar la preparación y supervisión del baño de las piezas con los productos seleccionados, que incluyeron a: Combo Antiblu, Sinesto $B^{\circledR}$ y Wolsin $1221^{\circledR}$. Se consideró además un paquete por cada línea con Tribromofenol, antimancha de uso rutinario en ambas empresas (cuadro 1). En total se prepararon 250 litros de solución a la concentración deseada.

El baño se realizó en una tina diseñada para este tipo de ensayos, sumergiendo las tablas durante diez segundos y dejando escurrir otros diez. 
BOSQUE 25(3): 79-87, 2004

Ensayos de eficacia cuarentenaria de tres formulaciones de antimanchas comercializadas en Chile para un proceso...

\section{CUADRO 1}

Fungicidas (tratamientos) seleccionados.

Selected fungicides.

\begin{tabular}{|llc|}
\hline Producto & Ingrediente activo & Concentración \\
\hline $\begin{array}{l}\text { Combo Antiblu* } \\
\left.\text { (Antiblu 375 }{ }^{\circledR}+\text { Antiblu CC }^{\circledR}\right)\end{array}$ & $\begin{array}{l}\text { Cobre 8 hidroxiquinolato 3,75\% w/v } \\
\text { Clorotalonil 45\% w/v + carbendazima } \\
10 \% \text { w/v }\end{array}$ & $4-1 \%$ \\
\hline Wolsin FL 1221 & Propiconazol 2,7\%, Fenporpimorph & $2,5 \%$ \\
\hline Sinesto B ${ }^{\circledR}$ & Di-4\%, ácido bórico 9\% & \\
& cuaternario & $8 \%$ \\
\hline Testigo 1 (sin producto) & & $10 \%$ \\
\hline Tribromofenol (Testigo 2) & Tribromofenol 98-99\% & \\
\hline
\end{tabular}

*Combo Antiblu es una mezcla de Antiblu 375 al 4\% y Antiblu CC al 1\%.

A medida que se fue armando el paquete respectivo, se numeró cada tabla, y una vez enzunchado, marcado con pintura spray.

Medición: En el horizonte tiempo del ensayo se realizaron tres mediciones en cada línea de acción que ocurren respectivamente: la primera, luego del acopio inicial al cabo de los 7 y 37 días respectivamente en cada línea; la segunda, luego del transporte marítimo después de los 52 y 82 días, y la tercera medición, al momento de llegar al destino final al cabo de los 79 y 109 días (figura 1).

La medición consistió en la revisión visual de cada una de las 80 tablas centrales de cada paquete, determinando el grado de ataque según la escala que se presenta en el cuadro 2 . Se consideró que las tablas de la periferia estaban sujetas al efecto borde, por lo que se descartaron.

\section{RESULTADOS Y DISCUSION}

Condiciones ambientales: En la Octava Región, la humedad relativa se mantuvo fluctuante entre $65 \mathrm{y}$, con mayor frecuencia, en $100 \%$. La temperatura ambiente se registra variable en un rango entre 5 y $22^{\circ} \mathrm{C}$ (figura 3 ).
CUADRO 2

Escala de medición de mancha y mohos. Stain and mould measuring scales.

\begin{tabular}{|cc|}
\hline Grado & Superficie manchada \\
\hline 0 & $0 \%$ \\
1 & $1-10 \%$ \\
2 & $11-25 \%$ \\
3 & $26-50 \%$ \\
4 & $>50 \%$ \\
\hline
\end{tabular}

En la Décima Región, la situación fue diferente. Las temperaturas no sobrepasaron $\operatorname{los} 14^{\circ} \mathrm{C}$, siendo el valor más bajo de $2,3^{\circ} \mathrm{C}$. La humedad relativa se mantuvo entre 80 y $100 \%$. Adicionalmente, durante el período se registraron intensos episodios de lluvia, lo que condicionó la alta humedad relativa presente que, aunada a las bajas temperaturas, habría propiciado la menor presencia de mancha y mohos en la madera, en comparación con la de la Octava Región (figura 4).

Debido a las características del ensayo que se plantea no debe haber mancha ni mohos en la madera, por lo tanto, ninguno de los productos cumplió con los requisitos planteados, ya que 
BOSQUE 25(3): 79-87, 2004 Ensayos de eficacia cuarentenaria de tres formulaciones de antimanchas comercializadas en Chile para un proceso...

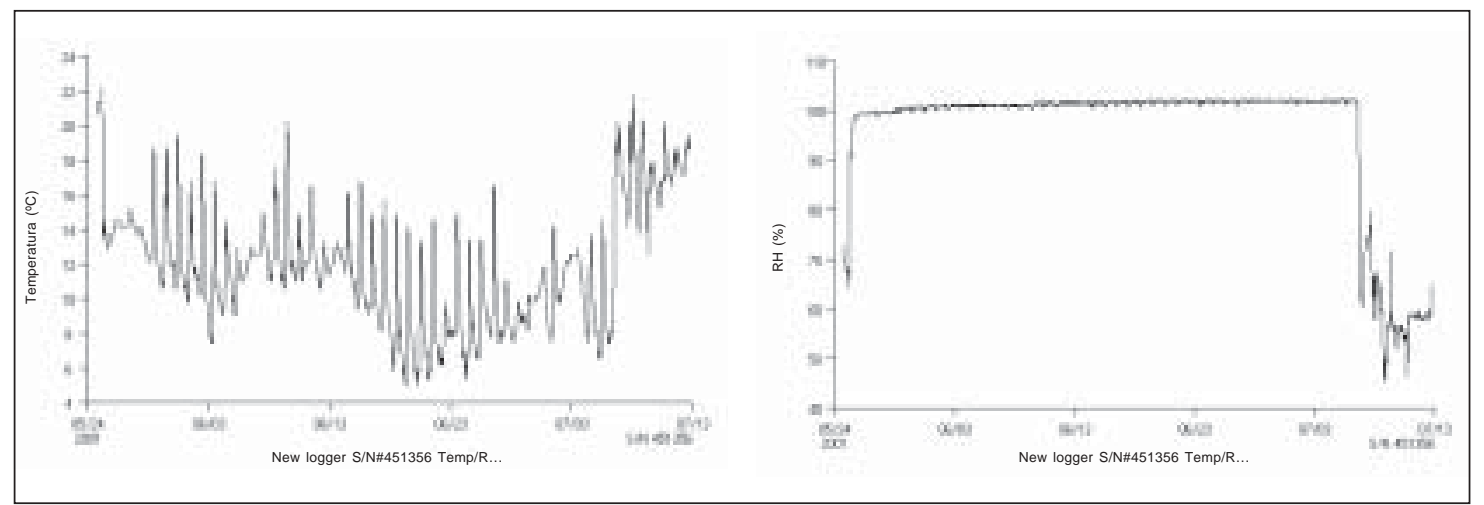

Figura 3. Registros climáticos. A: temperatura, B: humedad Octava Región.

Weather records. A: Temperature, B: Humidity, Eighth Region.

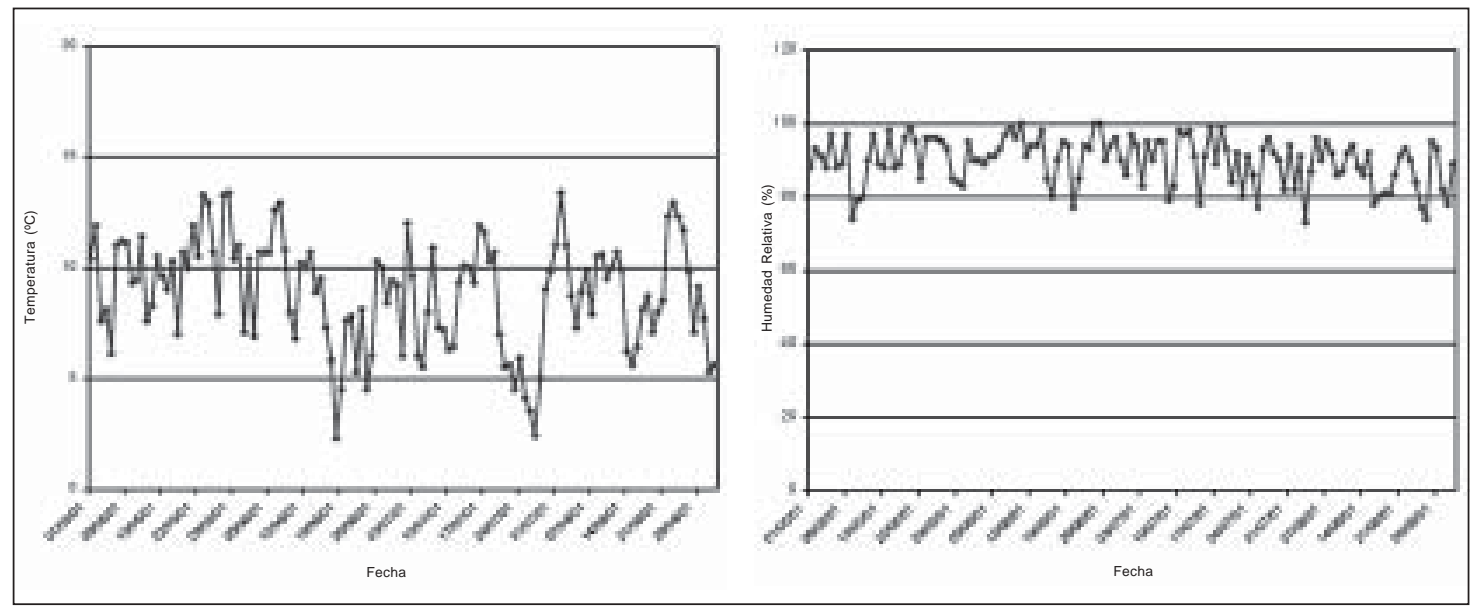

Figura 4. Registros climáticos. A: temperatura, B: humedad. Décima Región.

Weather records. A: Temperature, B: Humidity, Tenth Region.

todos presentaron mancha en mayor o menor grado; a pesar de ello, fue importante destacar cuál fue el comportamiento de los productos en las dos Regiones estudiadas.

Respecto de la eficacia de los productos, se observa que en la Décima Región éstos lograron un mejor rendimiento que en la Octava, al presentar menor porcentaje y grado de ataque, según escala, del hongo manchador (figura 5). Si bien los dos ensayos fueron de iguales características, hubo un desfase de un mes entre los empezados en la Octava y Décima Región. Las condiciones medioambientales de ambas regiones difieren en cuanto a sus características de temperatura y humedad. En la Décima Región llovió durante todo el ensayo, por lo cual la madera se encontraba saturada de agua, lo que se tradujo en menor desarrollo tanto de mohos como de manchas. En la Octava Región las condiciones medioambientales y de la madera fueron más favorables al desarrollo de mancha y mohos; así lo demuestran los resultados de los testigos que en la Décima Región no lograron el $100 \%$; es más, la línea 1 no alcanzó el 30\%, y la línea 2 sólo un 75\%. En cambio, en la Octava Región sí se alcanzó un $100 \%$ en las dos líneas a partir de la segunda medición en la línea 1 y primera medición en la línea 2.

Según Keirle (6) el riesgo de infección fúngica varía estacionalmente, siendo más severo en los 


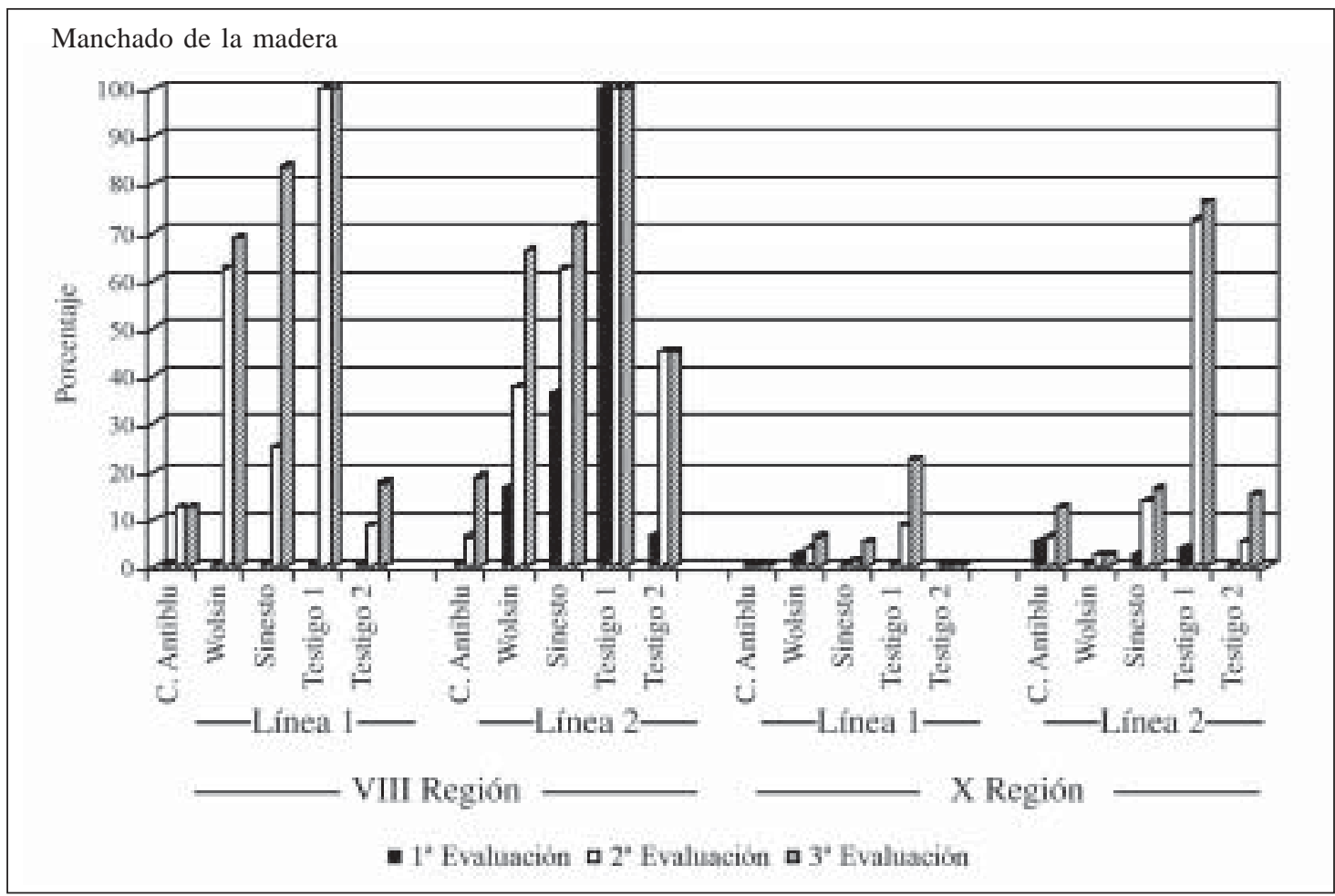

Figura 5. Relación de tablas afectadas en el período del ensayo.

List of affected boards during the test period.

meses de primavera-verano y otoño, durante el invierno el riesgo es menor. En términos generales, los hongos de la mancha azul requieren de algunas condiciones básicas necesarias para desarrollarse, como son nutrientes, humedad, oxígeno y temperatura favorables (7). Por lo tanto, el desarrollo de la mancha dependerá de la forma en que se encuentren las condiciones para el desarrollo de los hongos.

De los productos ensayados Combo Antiblu resultó ser el que mejor protegió la madera, en las dos Regiones, ya que al término del ensayo presentó entre un 10 a $20 \%$ de tablas afectadas en grado 1 (cuadro 3), lo que significa que cada tabla no presentaba más allá del $10 \%$ de su superficie manchada. Se observó también que el tratamiento con Tribromofenol a largo plazo no proporcionó una adecuada protección a la madera al presentar, en la Octava Región principalmente, mancha y mohos, también en grado 1 a 2 al cabo de 109 días, período de tiempo más largo al que estuvieron expuestos los paquetes.

En el caso de los agentes involucrados, en ambas Regiones se consideró de interés, además de la mancha azul, evaluar visualmente los mohos, ya sean blancos o verdes (Trichoderma sp.) desarrollados superficialmente sobre la madera, constatando que la mancha prevalece sobre los mohos.

Los paquetes expuestos por más tiempo (109 días), en ambas Regiones y para los tres productos en estudio, presentaron mayor grado de ataque de mancha que de Trichoderma sp. A mayor tiempo de exposición, la madera está más expuesta al manchado; por lo tanto, los productos antimanchas debieran proporcionar una protección de ésta por lo menos de 3-6 meses según Montes (4).

Cabe destacar que los resultados presentados se hicieron a base de número de tablas afectadas; esto significa que en una misma tabla podían coexistir tanto el hongo de la mancha azul como Trichoderma o mohos blancos, de allí que el porcentaje en algunos casos sea mayor de $100 \%$.

En la Décima Región también la mancha prevalece sobre Trichoderma sp. y los mohos blancos en baja proporción (grado 1), debido a que las condiciones ambientales y de la madera favorecieron una mejor protección a ésta (cuadro 4). 


\section{CUADRO 3}

Tablas afectadas por mancha y mohos por grado y agente en la Octava Región. Boards affected by stains and moulds, according to degree and agent in Eighth Region.

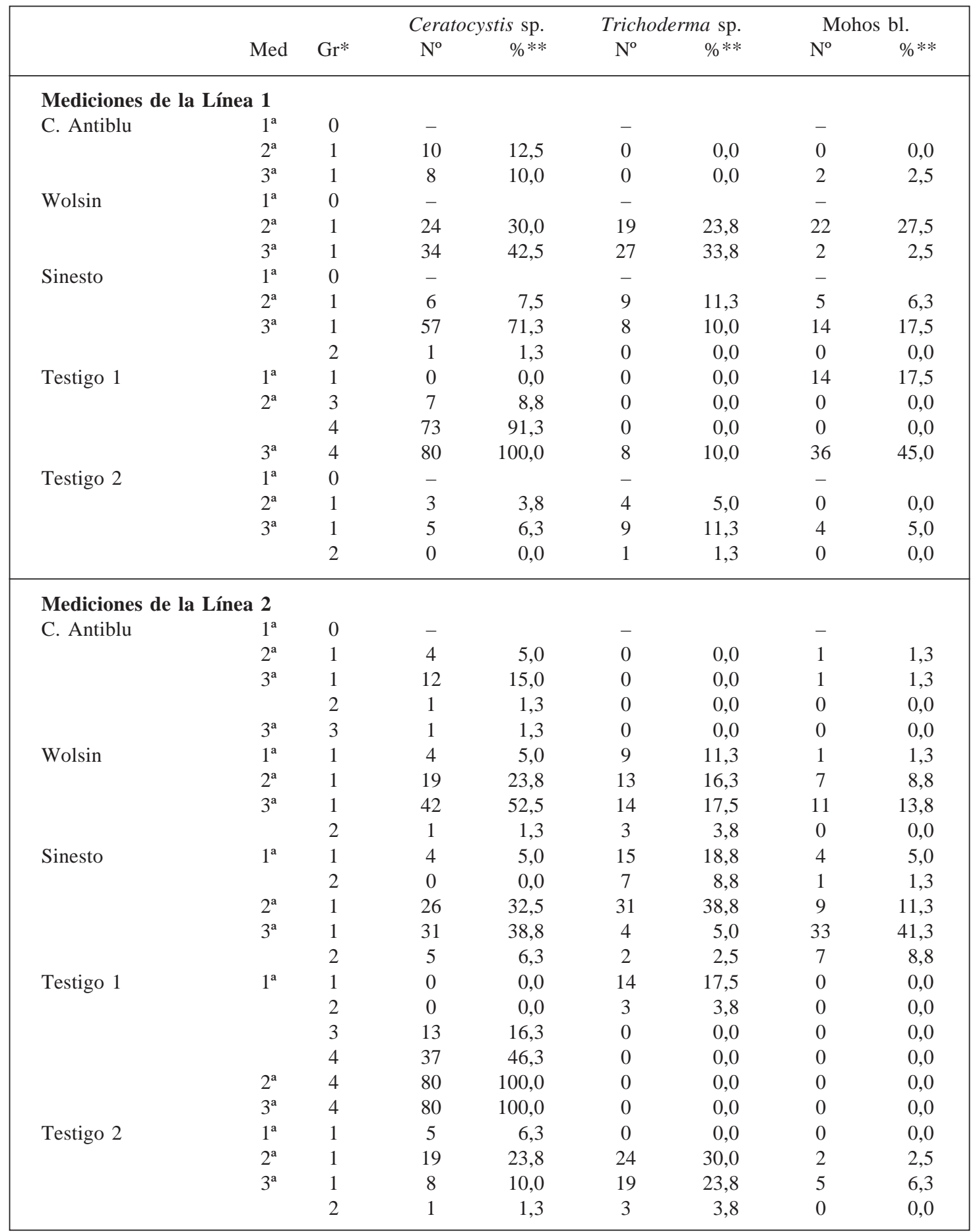

* Grado de ataque según escala, ** Una tabla puede presentar más de un tipo de hongo en distinta proporción. 


\section{CUADRO 4}

Tablas afectadas por mancha y mohos por grado y agente en la Décima Región. Boards affected by stains and moulds, according to degree and agent in Tenth Regions.

\begin{tabular}{|c|c|c|c|c|c|c|c|c|}
\hline & \multirow[b]{2}{*}{ Med } & \multirow[b]{2}{*}{$\mathrm{Gr}^{*}$} & \multicolumn{2}{|c|}{ Ceratocystis sp. } & \multicolumn{2}{|c|}{ Trichoderma sp. } & \multicolumn{2}{|c|}{ Mohos bl. } \\
\hline & & & $\mathrm{N}^{\mathrm{o}}$ & $\% * *$ & $\mathrm{~N}^{\mathrm{o}}$ & $\% * *$ & $\mathrm{~N}^{\mathrm{o}}$ & $\% * *$ \\
\hline \multicolumn{9}{|c|}{ Mediciones de la Línea 1} \\
\hline \multirow[t]{2}{*}{ C. Antiblu } & $2^{\mathrm{a}}$ & 0 & - & & - & & - & \\
\hline & $3^{\mathrm{a}}$ & 0 & - & & - & & - & \\
\hline \multirow[t]{2}{*}{ Wolsin } & $2^{\mathrm{a}}$ & 1 & 3 & 3,8 & 0 & 0,0 & 0 & 0,0 \\
\hline & $3^{\mathrm{a}}$ & 1 & 5 & 6,3 & 0 & 0,0 & 0 & 0,0 \\
\hline \multirow[t]{2}{*}{ Sinesto } & $2^{\mathrm{a}}$ & 1 & 1 & 1,3 & 0 & 0,0 & 0 & 0,0 \\
\hline & $3^{\mathrm{a}}$ & 1 & 4 & 5,0 & 0 & 0,0 & 0 & 0,0 \\
\hline \multirow[t]{3}{*}{ Testigo 1} & $2^{\mathrm{a}}$ & 1 & 6 & 7,5 & 0 & 0,0 & 1 & 1,3 \\
\hline & $3^{\mathrm{a}}$ & 1 & 17 & 21,3 & 0 & 0,0 & 2 & 2,5 \\
\hline & & 2 & 1 & 1,3 & 0 & 0,0 & 0 & 0,0 \\
\hline \multirow[t]{2}{*}{ Testigo 2} & $2^{\mathrm{a}}$ & 0 & - & & - & & - & \\
\hline & $3^{\mathrm{a}}$ & 0 & - & & - & & - & \\
\hline \multicolumn{9}{|c|}{ Mediciones de la Línea 2} \\
\hline \multirow[t]{2}{*}{ C. Antiblu } & $2^{\mathrm{a}}$ & 1 & 5 & 6,3 & 0 & 0,0 & 0 & 0,0 \\
\hline & $3^{\mathrm{a}}$ & 1 & 10 & 12,5 & 0 & 0,0 & 0 & 0,0 \\
\hline \multirow[t]{2}{*}{ Wolsin } & $2^{\mathrm{a}}$ & 1 & 2 & 2,5 & 0 & 0,0 & 0 & 0,0 \\
\hline & $3^{\mathrm{a}}$ & 1 & 2 & 2,5 & 0 & 0,0 & 0 & 0,0 \\
\hline \multirow[t]{2}{*}{ Sinesto } & $2^{\mathrm{a}}$ & 1 & 8 & 10,0 & 0 & 0,0 & 3 & 3,8 \\
\hline & $3^{\mathrm{a}}$ & 1 & 10 & 12,5 & 0 & 0,0 & 3 & 3,8 \\
\hline \multirow[t]{3}{*}{ Testigo 1} & $2^{a}$ & 1 & 49 & 61,3 & 2 & 2,5 & 13 & 16,3 \\
\hline & $3^{\mathrm{a}}$ & 1 & 38 & 47,5 & 8 & 10,0 & 8 & 10,0 \\
\hline & & 2 & 14 & 17,5 & 0 & 0,0 & 4 & 5,0 \\
\hline \multirow[t]{2}{*}{ Testigo 2} & $2^{a}$ & 1 & 4 & 5,0 & 0 & 0,0 & 0 & 0,0 \\
\hline & $3^{\mathrm{a}}$ & 1 & 12 & 15,0 & 0 & 0,0 & 0 & 0,0 \\
\hline
\end{tabular}

*Grado de ataque según escala, ** Una tabla puede presentar más de un tipo de hongo en distinta proporción.

Finalmente, con respecto a la presencia o no de separadores entre las tablas que conformaban los paquetes, no se encontraron diferencias prácticas entre uno y otro. El número de tablas afectadas con y sin separadores fue casi igual, y si hubo diferencias éstas fueron mínimas; por lo tanto, no se puede concluir que lo uno o lo otro fuera lo mejor.

\section{CONCLUSIONES}

- Al ser éste un ensayo de tratamiento cuarentenario en que no debe haber mancha en la madera, ninguno de los productos cumplió con los requisitos planteados.

- Combo Antiblu fue el más efectivo en ambas Regiones, a pesar que los paquetes bañados con este producto se mancharon en baja proporción.

- En ambas Regiones se desarrolló principalmente la mancha azul y Trichoderma sp.

- En relación al uso de paquetes empalillados no se observaron diferencias claras al respecto.

- Las condiciones medioambientales de la Décima Región propiciaron una mejor protección a la madera. 
BOSQUE 25(3): 79-87, 2004 Ensayos de eficacia cuarentenaria de tres formulaciones de antimanchas comercializadas en Chile para un proceso...

\section{AGRADECIMIENTOS}

Los autores expresan su agradecimiento al señor Jaime Vargas $C$. por su valioso aporte durante la realización del escrito.

\section{BIBLIOGRAFIA}

(1) INSTITUTO FORESTAL (CHILE). Estadísticas forestales. 2001 (citado el 10 de diciembre de 2002). Disponible en: <http://www.infor.cl>

(2) FERRELL, R., S. DUNCAN, S. RAM KAY, E. HADAR, Y. HADAR, R. BLANCHETTE, T. HARRINGTON, D. McNEW. Causes of Saptain in New Zealand. In KREBER, B. Strategies for Improving Protection of Logs and Lumber. New Zealand. FRI Bulletin 204. 1997. p. 25-31

Recibido: 11.03 .03

Aceptado: 14.11 .03
(3) AMERICAN SOCIETY FOR TESTING AND MATERIALS. ASTM D 4445-91. Standard Method for Testing Fungicides for Controlling Sapstain and Mold on Unseasoned Lumber (Laboratory Method). Annual Book of ASTM Standards. 1996. 4p.

(4) MONTES, P., H. PEREDO, D. LANFRANCO, S. IDE, H. DÖLZ. Una revisión de los productos alternativos al Pentaclorofenato de sodio y Bromuro de metilo utilizados en el sector forestal. Bosque (Chile), 2001, vol. 22 , $\mathrm{N}^{\circ} 1$, p. 85-93.

(5) ARRIAGADA, J., M. ZUNIGA Metodología para la evaluación técnico-económica de productos fungicidas. Tesis Ingeniería en Ejecución Forestal, Universidad de Concepción, 1996, 51 p.

(6) KIERLE, R. M. Effect of storage in different seasons on sapstain and decay of Pinus radiata D. Don in N.S.W Aust. For. (Australia), 1978, vol. 41, No 1, p. 29-36.

(7) SEIFERT, K. Sapstain of commercial lumber by species of Ophiostoma and Ceratocystis. In: M. J. WINGFIELD, K. A. SEIFERT, J. F. WEBBER. Ceratocystis and Ophiostoma Taxonomy, Ecology, and Pathogenicity. St. Paul (MN), USA. APS Press. 1993. p. 141-151. 